\title{
Learning in and from projects: The learning modes and a learning capability model
}

Production Planning and Control Journal

Eltigani, Adil; Skema Business School, Paris campus, Innovation Academy, France adil.eltigani@skema.edu

Gardiner, Paul; SKEMA Business School, Université Côte d’Azur, Lille, France paul.gardiner@skema.edu

Kirkham, Richard; School of Engineering, Department of Mechanical, Aerospace and Civil Engineering, The University of Manchester, UK richard.kirkham@manchester.ac.uk

Williams, Terry; University of Hull, Hull University Business School, UK terry.williams@hull.ac.uk

Ou, Lixiong; Northwestern Polytechnical University, China lixiong@nwpu.edu.cn

Calabrese, Antonio; Politecnico di Milano, Italy calabrese@mip.polimi.it 


\begin{abstract}
The notion of 'project delivery' is well embedded in and across the management and organisational sciences literature - generating a narrative that reflects and recognises the instrumental nature of projects and programmes in strategy execution. Project management, as a distinct and well-established body of research enquiry, has increasingly sought to focus our attention on the impacts of complexity, risk and uncertainty in projects; the corollary being a desideratum to strengthen our theoretical understanding of how insight and learning from projects may influence improvements to organisational efficiency. The wider literature suggests that organisational learning remains a challenging proposition, particularly in the context of organisations operating in environments of high complexity. In this paper, we enhance the conversation on organisational learning through a series of case studies, generating evidence of thirteen 'learning modes'. The paper proposes that mature organisations tend to exhibit a greater number of learning modes and that there is a tendency to capture and socialise knowledge with a greater emphasis on the context of the learning situation rather than the learning artefact in isolation. The empirical evidence gathered in this paper forms the basis of a capability model, characterised by the thirteen modes of learning. The model intimates that learning occurs, and is more effective, when knowledge and information are enacted in practice through the learning modes which form a nucleus of the organisational learning capability. The research concludes with a call to action that emphasises the strategic importance to improve learning practices in project orientedorganisations.
\end{abstract}

\title{
Keywords
}

Learning; knowledge management; learning capability; modes of learning; dynamic capabilities; structuration; practice 


\section{Introduction}

Projects and programmes are unique, impermanent actions typified by varying degrees of risk, uncertainty and complexity; these characteristics in themselves suggest that projects may not, in isolation, provide useful antecedents for organisational learning (see Rolstadas (1994)). In this paper we argue that project oriented organisations should seek to develop 'learning capability’ in their routines and practices as a means of developing new capabilities, encouraging innovation and maintaining financial sustainability. Thus, the desideratum to connect project learning to wider organisational practices such as strategy development, performance management, risk and knowledge management and corporate governance management practices is proposed.

Learning from projects and project management practice continues to deliver a rich vein of academic enquiry in the literature yet the majority of these endeavours adopt a ‘sender/receiver’ approach (Hartmann \& Dorée, 2015) - even where a systemic organisationwide approach is taken to learning (e.g. Duffield and Whitty, 2015). McClory et al. (2017) conceptualise the lessons-learned process by demonstrating how knowledge gathering methods fit within organisational routines; in this paper we advance those propositions by arguing that knowledge transfer and learning is a complex process, irreducible to simple material or signal transfer.

Our intentions lie in the domains of knowledge management, learning and dynamic capabilities. These lenses enable us to build on practice theories and in particular structuration theory (see Giddens, 1984) and advance previous studies on organisational learning in the context of project studies (see Soderlund (2010), Ahern, Leavy and Byrne (2014a, b) and Davies \& Brady, (2016)). Our conceptual framework seeks to connect the organisation-wide structure, including organisational capabilities, resources and knowledge assets with the micro-level activities and 'episodes of learning', leading to the extension and creation of new 
knowledge and capabilities and ultimately, enhanced business value. Thus, the objectives of this research are:

1. To investigate the modes of learning in projects and programmes across a sample of organisational settings, and

2. To investigate the learning mechanisms (modes) observed in those project-based organisations that may lead to successful project capability development

We draw on data garnered from participant organisations situated in Europe, the United States, Asia and the Middle East. The results uncover thirteen modes of learning from which, a learning capability model is proposed, representing a continuous learning-in-practice phenomenon that is enduring throughout the project lifecycle. This model describes a metacapability and not only a process, in that it encompasses resources and behaviours in addition to processes.

\section{Learning and Knowledge Management}

It is entirely plausible that organisations perceive knowledge creation as fundamental to achieving and sustaining competitive advantage. A range of perspectives in the extant literature explore the mechanisms and processes associated with knowledge creation, utilisation and transference. The traditional view classifies knowledge into distinctive and separable components: the first being explicit knowledge that is relatively simple to codify

and transfer, the second being the more complex (arguably ephemeral) tacit knowledge that is acquired through cognition and is difficult to codify and transfer (Polanyi, 1966, Nonaka, 1991). This view advanced a general understanding of knowledge and remains influential today, particularly in practice but also in research (Zhu, 2006, Tsoukas, 1996). This taxonomic perspective of knowledge (Tsoukas, 1996) builds on a positivist epistemological 
position and overlooks the complex social side (Easterby-Smith and Prieto, 2008). However, some critics argue that this taxonomy does not provide a full understanding of the mechanics of knowledge (Seely and Duguid, 2001, Orlikowski, 2002, Tsoukas, 1996, Tsoukas, 2009). Others view knowledge as a transferrable substance, for instance, Lin et al. (2005) develop a framework for knowledge transfer from the sender to the receiver through a medium; this is similar to signal transmission in telecommunications. We argue that knowledge transfer and learning are more socially complex processes that cannot be reduced to material or signal transfer.

An alternative stream of thought 'sought to understand the nature of organizational knowledge through making analogies between organizations and the human brains on the one hand, and the organizations and the individual minds on the other' (Tsoukas, 1996, p. 13). Here the different types of knowledge (tacit and explicit) are seen as linked together and are inseparable. Tsoukas (1996) argues that tacit knowledge is inseparable from other forms of knowledge and that it 'is not made up of discrete beans which may be ground, lost or reconstituted.' (p.14), rather it is the basis for all types of knowledge, and that explicit knowledge always draws on a tacit component.

Tsoukas (1996) argues that 'no set of rules can ever be self-contained, complete. Thus we are led to the conclusion that every act of human understanding is essentially based on an unarticulated background of what is taken for granted' (p. 16). Orlikowski (2002, pp 249-273) builds on this view to develop the concept of knowing in practice arguing that knowledge is not static but 'an on-going social accomplishment, constituted and reconstituted as actors engage the world in practice’ (Orlikowski, 2002, p, 249). For her, this reciprocal relationship between knowledge and practice implies the necessity to investigate both together, as she puts it: 'It suggests there may be value in a perspective that does not treat these as separate or separable’ Orlikowski (2002, p. 250). Seely and Duguid (2001) also suggests that the practice 
perspective solves the paradox of the knowledge dichotomy of what they refer to as sticky and leaky knowledge. Ollus et al. (2011) argue that key stakeholders need to jointly participate in learning process to ensure collaborative alignment and to have common understanding throughout the project.

Nonaka (1991) promotes the distinction between explicit and tacit knowledge, this is broadened in Nonaka and Toyama (2003) to an alternative theory, drawing upon Giddens’ 1984 concept of the duality of agents and structures. In this alternative theory, Nonaka and Toyama argue that knowledge creation is a 'dialectic process where new boundaries are created through the dynamic interaction between the agents as well as between agents and structure' (p. 9). Similarly, Tsoukas (2003) argues that tacit knowledge can only be observed when it is enacted and put into practice. He explains that 'tacit knowledge cannot be 'captured', ‘translated', or ‘converted’ but only displayed and manifested, in what we do'. Similarly, Al-Busaidi and Olfman (2017) argue for the importance of considering 'human factors’ as they directly and significantly affect knowledge sharing.

In the previous paragraphs, we discuss the trends in organisational knowledge literature and the recent developments in linking knowledge-to-practice theories. Some authors have also addressed organisational knowledge and its relationship to other phenomena, such as dynamic capabilities - with its origins in the resource based view of strategy. For example, EasterbySmith and Prieto (2008) show that the literature on knowledge management and dynamic capabilities has acknowledged their symbiotic relationship, in that the creation and development of dynamic capabilities actually lies in the ability to create and transfer knowledge. Winter (2003) and Zollo and Winter (2002) argue that dynamic capabilities can be developed through the process of deliberate learning activities. Similarly, Nielsen (2006) identifies knowledge management activities that support the development of dynamic capabilities. He argues that the concept of dynamic capabilities can be understood from within 
knowledge management activities and advises that managers need to focus on knowledge management in order to operationalise dynamic capabilities.

Although project management has developed in last few decades and has become common practice in organisations, research shows that there is usually limited learning from doing projects (Newell and Edelman, 2008). Typically, projects are characterised as temporary, multidisciplinary initiatives with unique outcomes. Leaning in projects is influenced by external factors such as the time bound nature of projects, where emphasis is on meeting deadlines rather than on developing long-term project management capabilities (Mainga, 2017). An important research study on learning in project management is the empirical work of Prencipe \& Tell (2001) resulting in the notion of three broad 'landscapes' for learning: explorer, navigator and exploiter which respectively draw on communication between people, basic information technology and advanced information technology. Their work builds on Zollo and Winter (2002) where the argument is that dynamic capabilities are shaped by three learning mechanisms: first is the experience accumulation, second knowledge articulation and third is knowledge codification. Zollo and Winter propose that experience accumulation is a semiautomatic process that is established through 'deliberate investments in knowledge articulation and codification activities' (page 339). In their view, all three learning mechanisms must exist and interact in practice to build new capabilities. In another study, Newell and Edelman (2008) refer to learning in projects and the ability to transfer knowledge as a dynamic capability, since it is concerned with changing the current routines.

From the above discussion, we infer the following three premises:

1. Knowledge can only be observed and studied from within the practice in which it is instantiated. 
2. Agents add their own understanding and knowledge to their actions when they follow rules or knowledge that is codified.

3. Learning is essential in the process of developing organisational capabilities.

Premise 1 includes all types of knowledge and argues that knowledge can only be observed in action. Premise 2 reinforces this point by arguing that what is traditionally referred to as explicit knowledge is non-static in nature; rather individuals add their different knowledge bases to it and hence they may have different perceptions about it and hence act differently.

\section{The Conceptual Framework}

In order to sufficiently investigate patterns of learning in project management and the mechanisms that lead to development of project management and organisation capabilities, a conceptual framework based on theories of practices is required. The framework allows scrutiny of the social structure (Giddens, 1984) of the organisation and the activity configurations (Regnér, 2008) allowing the possibility to investigate how learning happens and how organisations reconfigure their practices based on the results of learning.

The initial theoretical position of this research builds on the work of Easterby-Smith and Prieto (2008) and the structuration theory of Giddens (1984). Easterby-Smith and Prieto (2008) propose that learning is considered as the 'central mechanism' that links dynamic capabilities and knowledge management in an organisational context. This conceptualisation is useful in providing a theoretical basis to the pilot-study. Results from the pilot study indicated the need for further research on the learning mechanisms that lead to the development of dynamic capabilities. For the purposes of more fully understanding those learning mechanisms, a revised conceptual framework was developed, as presented in the following paragraphs, which builds on the concepts found in the theory of structuration 
developed by Giddens (1984), especially those concepts relating to the characterisation of agents as being knowledgeable and reflexive.

Giddens (1984) considers social practices as the main driver and observable unit in social science where he argues that 'the basic domain of study of the social sciences, is neither the experience of the individual actor, nor the existence of any form of social totality, but social practices ordered across space and time’ (Giddens, 1984). In the modified theoretical framework we separate, for analytical purposes, between agents (knowledgeable and reflexive) and the prevailing social structures. The research framework also builds on the concept of activity configurations developed by Regnér (2008).

Regnér (2008) compares and contrast the strategy-as-practice approach with the dynamic capabilities perspective and how they can complement each other. The root of strategy-aspractice is found in social theories (e.g. Giddens, 1984 and Bourdieu, 1977) with the main intent of looking at strategy formulation and formation as an ongoing social practice (strategizing). Whereas, the dynamic capabilities perspective is rooted in evolutionary economics and is concerned about the firm level performance. Regnér (2008) develops a framework with 'activity configurations' as the unit of analysis and illustrates that 'activity configurations that involve specific combinations of certain actors, socio-cultural contexts, cognitive frames, artefacts and structural properties, besides diverse practices, are a more useful unit of analysis since they emphasize the significance of inter-linkages and interdependencies among these in the process of strategy formation over time'. In the framework, Regnér (2008) separates the social structure of the organisation from agents and treats 'activity configurations' as an interaction between the two. This view was central in the development of our theoretical framework, as the research takes both a micro-practice and a system-wide perspective. This helps investigate the learning mechanisms at the temporary project level and their interaction with the permanent organisation. 
In the following section, we discuss the theoretical background of the research followed by discussion of the proposed conceptual framework and how it was informed by theory from the available literature.

The proposed framework suggests a practice perspective. The central intent is to separate, for analytical purposes, the structural properties (rules and resources) that govern the activities of doing projects and the related organisational activities in wider sense (such as strategy) from the actual actions of actors (learning and reconfiguration); in other words to distinguish between what organizations have (structure and resources) and what people do. This is important for developing the research methodology and for guiding the data collection.

The framework is shown diagrammatically in Figure 1 below. The upper part of the diagram represents the tangible elements. These include the formal knowledge components (explicit) that are codified as organisational assets, the formal knowledge management system (if one exists), and the current capabilities of the organisation. In addition to this, the structure also includes all the resources that are possessed by or available to the organisation from external sources.

The lower part of this framework contains the activity configurations (Regnér, 2008). The argument here is based on the first premise that knowledge can only be observed and studied from within the practice in which it is instantiated; the agents' knowledgeability and motivation for action (Giddens, 1984) are placed within the activity configuration, as a process of learning and reconfiguration of practices. This reflects the second premise introduced earlier. 


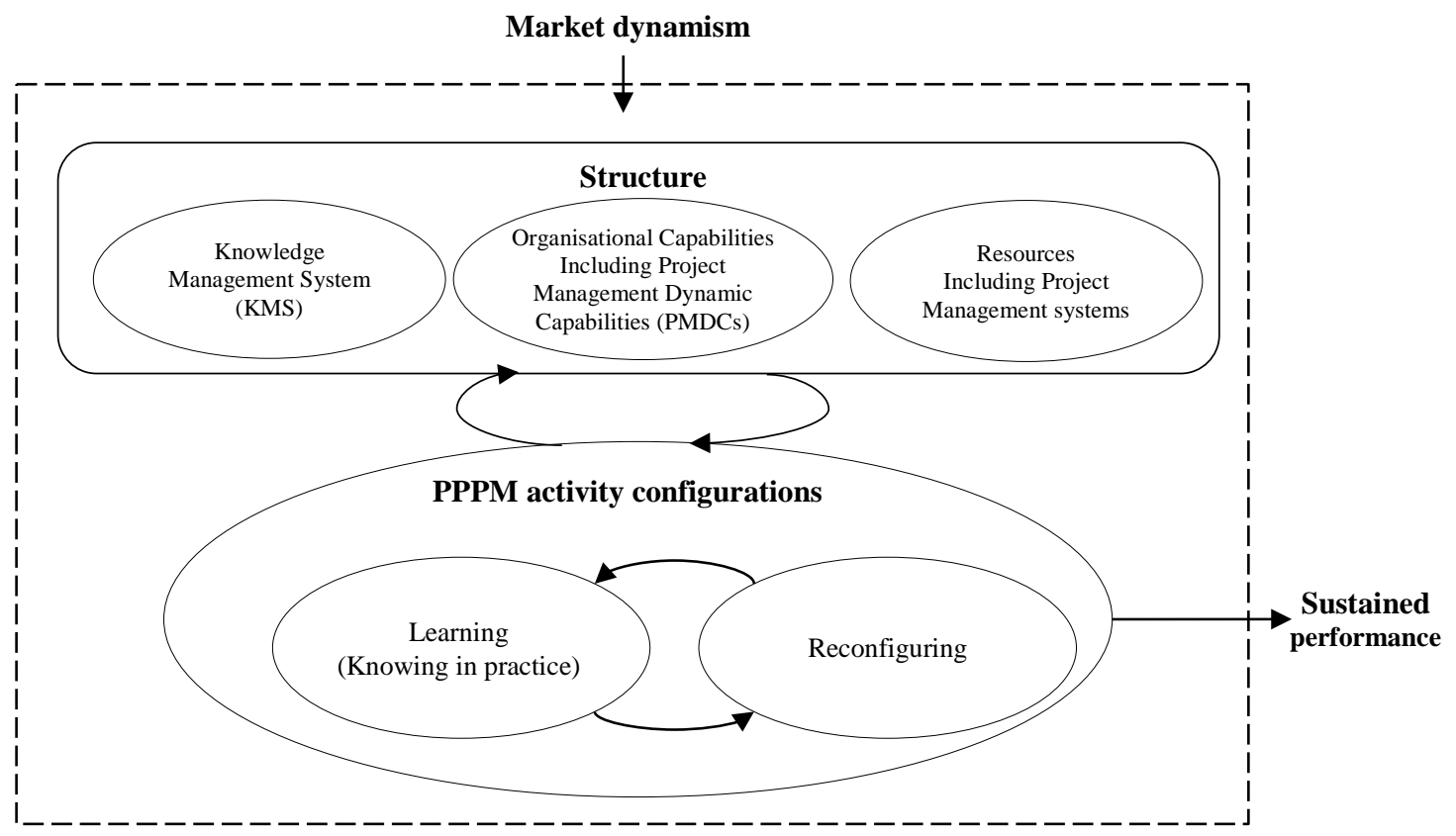

Figure 1- Conceptual framework

The lens of investigation draws upon Giddens's structuration theory (1984); the actions are outlined as activity configurations (see Regnér, 2008), implying a collection of actions that coalesce to form an observable set of activities that may be witnessed (and analysed) by the researcher. These activities represent project management practices (and routines) and related activities such as strategy formulation, portfolio management and organisational knowledge management. In this paper we argue for the connection of project management to the wider organisational activities and establish a basis for a more holistic and systemic recognition of the inter-relationship of projects and the broader corporate environment (Grundy (1998), Morris and Jamieson (2004))

Each episode of an activity configuration represents a small increment of time, like a snapshot, encapsulating a set of activities resulting in change. The social structural properties include the organizational structure, polices, and procedures. Feedback from activity configuration episodes, can lead to learning, which initiates changes in future activity configurations, and also to changes in the structure. The empirical data supports the view that 
this iterative process of learning and change leads to the development of new organisational capabilities - reflecting premise three mentioned above.

The reverse arrows between the structure and activity configurations in Figure 1 represent this perpetual bi-directional feedback. It is referred to as the ‘duality of structure’ by Giddens (1984), signifying that the structural properties are both medium and outcome of the agents' activities. As opposed to Easterby-Smith and Prieto (2008), in this model the learning aspect and tacit knowledge are placed within the activity configurations part of the model. This accords with the perspective of 'knowing in practice' developed by Orlikowski (2002).

Following the above explanation of the conceptual framework, it is useful now to take a more detailed look at its two major parts:

1. Structure: This includes the rules, resources and dynamic capabilities. Another important aspect of the structure is the knowledge management as a system and explicit knowledge; whereas knowledge of how things are done in practice (traditionally referred to as tacit knowledge) is part of the activity configurations, as explained below. The reasoning for this is that tacit knowledge can only be observed when instantiated in action.

2. Activity configurations: Are the human actions within the organisation. They include the activities of actors in performing strategic and project management related activities - more specifically, they include the existing and newly reconfigured activities that are the outcomes of the interacting elements in the upper part of the diagram in Figure 1, which are primarily responsible for the reconfiguration and development of new capabilities required to meet changing environments. The activity configurations also include the process of learning or knowing in practice. The 
assumption here is that tacit knowledge is an active element of 'knowing' and is inseparable from action (Orlikowski, 2002).

The developed conceptual framework connects the organisation-wide structure including capabilities and resources with the activity configurations to allow investigation of learning patterns and mechanisms in project based organisations taking into consideration the organisation-wide practices.

However, it is important to remember that people do not always act in a rational way based on the rules of the social structure, but their actions can also be influenced by their motivation for action. For instance, Tommelein et al. (2014) build on Schiefloe (2011) to develop what they refer to as the "Pentagon model” for analysing project organisation performance. In the model, emphasis is on the formal and informal structures, social relations, networks and culture in project organisations and how they can shape actions and behaviours of actors. Tommelein et al. (2014) point out that social relations represent an informal structure that defines relationships among actors such as friendships, alliance and conflict. Whittington (2010) discusses the tendency of agency to follow one social system or do otherwise and argue "everybody has some sort of social power". In this sense, actions that follow the norms of the social system or otherwise will have consequences based on the prevailing rules and at the same time gradually change the social structure in a duality based interaction (Giddens, 1984).

This section has outlined the theoretical background to the research, briefly exploring the ontological and epistemological considerations that apply to our assumptions regarding the nature of knowledge and learning mechanisms within the project management and organisation-wide related activities. This explanation of the theoretical orientation of our research is supported by a discussion of the key literature from which it has been developed. 
The resulting conceptual framework for this research has then been described, with an explanation of its relationship to the literature. This framework was used to shape the methodology for our empirical research, which is outlined in the next section.

\section{Research Methodology}

The methodological approach seeks to complement the exploratory and inductive nature of the study. Our epistemological orientation draws predominantly from practice theories, and in particular, Giddens’ structuration theory (1984), which suggests that a micro-practice perspective while paying attention to the wider organisational activities is suitable and appropriate to the nature of the data.

The methodology is characterised by a qualitative case-based approach, applied through a ‘micro-practice’ lens. By taking both a micro-practice and a system-wide perspective the research benefits from the collective learning capacity across multi-organisational domains and levels. This approach lends itself to finding deeper and richer learning episodes than would be possible if a more narrow perspective was used. An analogy here would be the iceberg model used by Bosch et al (2013) to explain the study of evolutionary learning using a systems approach.

In comparison, traditional project management is historically limited to using variations of the familiar lessons learned process to enhance and improve project management practice (Thompson, 2005). However, research has shown that this approach has suffered from a lack of effectiveness (e.g. Shokri-Ghasabeh, \& Chileshe, 2014; Goffin et al., 2010) that may be related to its application within the relatively tight boundary that project management processes often operate in. Once we open up the process of learning to a more systemic analysis, greater opportunities for understanding issues and problems arise since our field of 
view increases to encompass more of the organisation. This allows issues of higher complexity, spanning organisational internal and external boundaries, to surface and creates additional awareness and richness. A more parochial view may still identify, address and fix the symptoms of a problem but without a deeper understanding of the broader systemic causes will be of temporary and limited value. This represents a lost opportunity for deeper root cause analysis. Accordingly, we have designed the methodology used in this research to span the learning in projects and related organisational activities in organisations in a bid to open up new avenues of analysis to allow the research to uncover systemic learning mechanisms and processes.

In the theoretical framework we propose activity configurations as the unit of analysis (Regnér, 2008), where the process of formation of (activity configurations) is influenced by the wider organizational conditions. This involves collective activities, including diverse actors from within, and external to, the organization drawn from the prevailing social structure. The expectation was that 'learning episodes' would reveal themselves within the activity configurations portrayed by the interviewees.

The methodology is underpinned by the conceptual framework and draws from the approaches developed by Regnér (2008) and Easterby-Smith and Prieto (2008). It was chosen to help with the identification of information to be collected and analysed (Miles and Huberman, 1994) and for the identification of complex and causal relationships. Yin (2009) is firm in the need for a theoretical proposition for case studies before the collection of any data. The case study interview protocols and database coding structure were created according to recommendations for establishing construct reliability and validity (Yin, 2009). More specifically the interview protocol design was guided by the structure and relationships in the conceptual framework. 
The research seeks to explore the role of learning capabilities and dynamic capabilities in the projects. Specifically, the researchers hope to shed light on how these capabilities interact with other capabilities of the organisation to facilitate balanced and dynamic changes that ultimately leverage competitive advantage. A qualitative approach is preferable to a quantitative approach: qualitative research (Creswell, 2017) enables identification of the contextual factors that relate to the phenomena of interest, facilitates the description of complex phenomena situated and embedded in specific contexts, and is useful for studying a limited number of cases in depth. It can also yield a much richer and more detailed picture.

A total of 47 interviews were collected from 23 organisations in 6 countries in Europe, the Middle East, Asia and North America. Organisations included a mixture of private and public sector organisations; large, medium and small-sized organisations. Data was collected from a variety of job roles, levels and projects in the organisations studied. The process of finding interviewees varied depending on the relationship established between case organisation and the researchers. For some organisations, the main contact helped to identify and get approval for a cross-section of people to be interviewed. For others, especially smaller organisations, it tended to be based on personal contacts and networks. This purposive selection of samples is important in qualitative research, especially where the target sample is small compared to the population (Miles and Huberman, 1994).

A limitation of this research and possible sources of error can be in the diverse nature of organisations studied belonging to diverse cultures and legal frameworks, which limits the characterisation and classification of organisations. Another limitation lies in our subjective classification or organisational maturity rather than using an attested maturity model.

\section{Data analysis}


In this section, we present the analysis of data. The interviews were transcribed and coded (using the Nvivo 11 software package) following the structure described in Table 1, which includes all variables found in the conceptual framework (Figure 1). Consequently, a series of data tables at an increasing level of abstraction were constructed as described below.

\section{Level 1: Data analysis tables}

The results generated from the interviews within each organisation are presented in a single table format; each represents a unique organisation and is based on a template structure (see Table 1) similar to the conceptual framework described in the previous section of this paper. By way of introduction, each table is accompanied by a brief description of the organisation and a brief summary of the key issues emerging from the interview data. The size and structure of each data analysis table reflects the number of interviews conducted and the richness of the data.

\begin{tabular}{|c|c|c|c|c|c|c|}
\hline \multicolumn{3}{|c|}{ Structure } & \multicolumn{2}{|c|}{ Activity Configuration } & \multicolumn{2}{|c|}{ Outcomes } \\
\hline $\begin{array}{c}\text { Knowledge } \\
\text { Management } \\
\text { System }\end{array}$ & $\begin{array}{c}\text { Dynamic } \\
\text { Capabilities }\end{array}$ & Resources & $\begin{array}{c}\text { Learning } \\
\text { (Knowing in } \\
\text { practice) }\end{array}$ & Reconfiguring & $\begin{array}{c}\text { New Dynamic } \\
\text { Capabilities }\end{array}$ & $\begin{array}{c}\text { New } \\
\text { Resources }\end{array}$ \\
\hline
\end{tabular}

Table 1: Template structure for the data analysis tables

\section{Level 2: Data analysis cards}

Following the initial data analysis, a second level of data examination and analysis was performed in order to identify specific learning episodes within each organisation; the purpose being to reveal learning practices that were enacted and the modes of capabilities, which the organisations developed as a result of these. A total of 60 data analysis 'cards', each one representing a discrete learning episode (activity configuration), were developed using the template shown in Table 2 


\begin{tabular}{|l|l|}
\hline \multicolumn{2}{|l|}{ Company code } \\
Few words description <e.g. Big multinational manufacturer $>$ \\
\hline Trigger & New resources \\
\hline Learning & Enabling/Dynamic capabilities \\
\hline Action/reconfiguration & New capabilities \\
\hline
\end{tabular}

Table 2: Template structure for data analysis cards

\section{Level 3: Summary analysis table}

A third level of analysis was performed, at a higher level of abstraction, in order to synthesise the emerging themes derived from the learning episodes identified across all 23 case-study organisations. The resulting analysis is presented in a summary table that considers: (i) the learning episode code, (ii) type of business, (iii) size of business, (iv) maturity of business, (v) trigger area, (vi) enabling/dynamic capability, (vii) locus of new capabilities, (viii) learning practices and (ix) learning modes. It is worth emphasising that it was the learning modes which held the most pertinent information associated with the objectives of the research. The end of the analysis identified a total of thirteen distinct learning modes. These are discussed at length in the next section, which gives rise to Level 4 of the data processing and analysis work.

\section{Level 4: Cross-case analysis}

An in-depth cross-case analysis completes the data processing and analysis work. In the crosscase analysis, each of four sets of learning mode is used as a basis of comparing the results of the different cases study organisations, with a reflection on the type of capabilities associated with each of them.

\section{Results and discussion}

\section{Learning modes}


As discussed in the previous sections, data was analysed to find learning practices that lead to new organisational capabilities or the modification of existing ones. From the data, we identified clear and discrete learning modes, which represent a summation of the learning practices identified in the research. Figure 2 represents the final set of thirteen learning modes discovered by this study. We do not suggest that this set is exhaustive as other modes of learning can still be discovered by further research in the future. Rather, they form the nucleus of the learning model (Figure 3) and are the active components of learning capability.

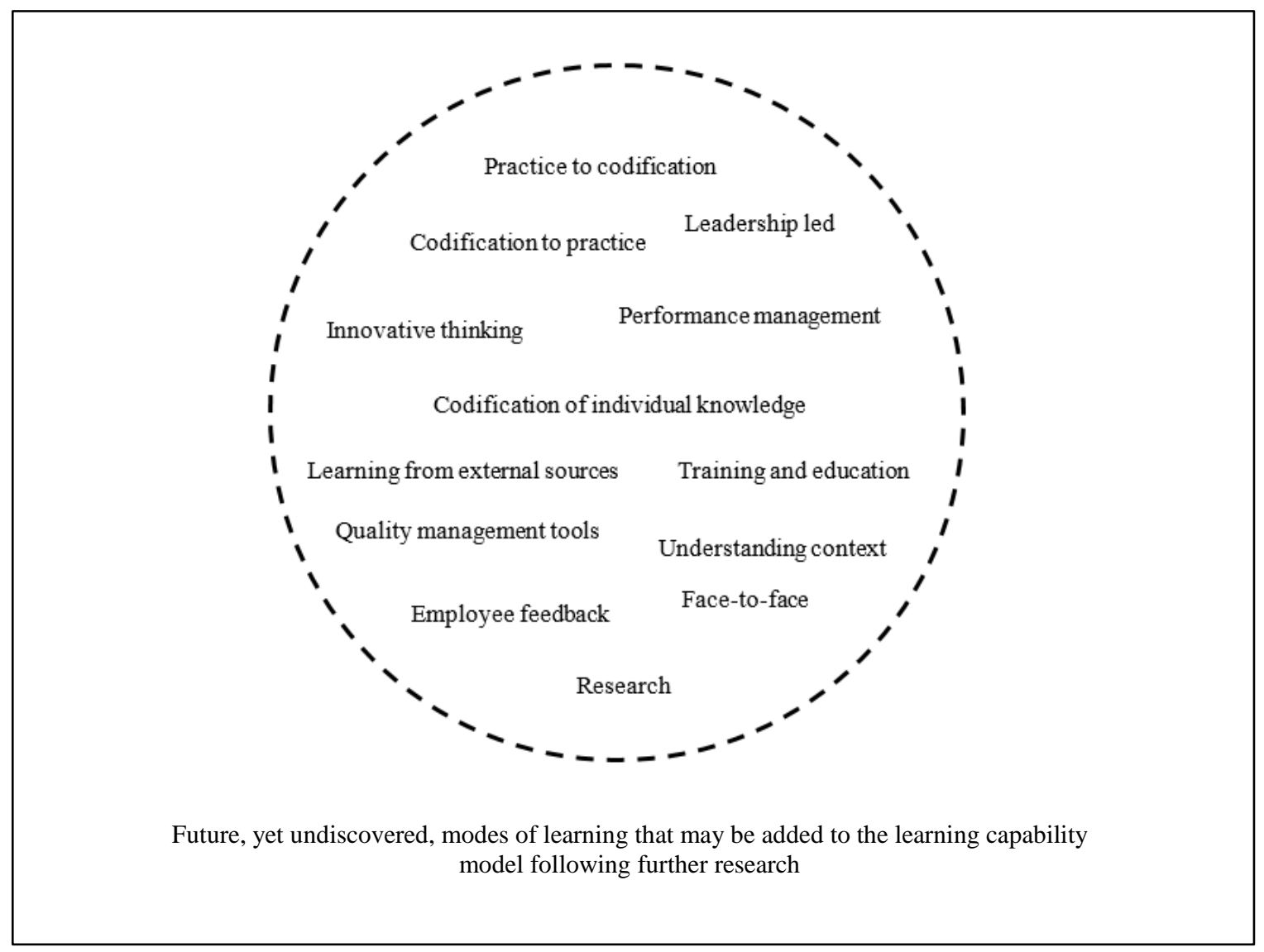

Figure 2: The final set of discovered learning modes

The first set of learning modes are related to codification of knowledge and how codified knowledge is used in practice. They are:

\section{Codification of individual knowledge}


This refers to the situations where the organisation realises the need to develop standardised processes, procedures, policies, etc. The data shows that, in this mode of learning, the organisation recruits qualified employees or uses existing employees to write the documents needed to establish standardised processes and policies. These documents are essentially codified artefacts of corporate knowledge, which are then embedded in the governance mechanism of the organisation at different levels. These learning artefacts shape and direct the behaviour and practices of employees. Data from interviews show that this mode of learning usually happens in less mature organisations.

\section{Practice to codification}

In this mode of learning, organisations deploy a range of tools and techniques to capture or codify lessons from routines, which can later be put into practice. The simplest way is similar to capturing tacit and explicit knowledge in classical knowledge management (KM) systems or by using the knowledge to develop or improve processes, procedures, policies, etc.

The data indicates that high maturity organisations also capture information about the context of situations and the social traits and personalities of the people involved. For example, capturing lessons learned within the context of the prevailing culture and affected people. For instance, a senior officer of a high maturity organisation one of the cases says about lessons learned:

We have them all [lessons learned] available, and we have them all tied to personalities that were available

Another manager declares:

Knowledge is contextual, it is about the situation, you can work a project effectively in one place and you can come to our organisation and you can fail because of the dynamics, the politics, the people and the nature of things are done differently. 
The data shows that this learning mode in its simplest form is followed by high maturity and moderately mature organisations, but high maturity organisations tend to place greater emphasis on the context of learning situation and the people involved. The type of capabilities developed as a result of this mode of learning span from document control to global project management and finding a balance between process rigor and fast decision making.

\section{Codification to practice}

As discussed in the first learning mode, codified or sanctioned knowledge can be in the form of a process or a policy developed by experienced or knowledgeable people for other people to read and follow in practice; in this way some of the knowledge is transferred from one to another. Another form of learning can be by way of transferring lessons learned and case studies collected from practice in a documented format (e.g. in a knowledge management system). The challenge here is how to transfer maximum knowledge, meaning how to maximise the throughput of learning from an existing or past situation, via documentation, to learning and onto improved future practice in a new location or context. Naturally, knowledge will fade when passing through each step in this knowledge transfer process.

The second set of learning modes is contextual learning which includes: understanding context and research.

\section{Understanding context}

Evidence from the interviews demonstrates that mature organisations are acutely sensitive to and aware of context; this mode of learning is dominant where organisations enter (or seek to enter) new markets and where they desire to appreciate the legislative and cultural landscape and how this may impact upon their strategy.

The interviews provide a useful example, where a senior manager opines the necessity to understand the cultural differences in the various offices of the company around the world: 
We always jog between the company spirit and at the same time trying to preserve the local culture. So this is a very difficult trade off to manage but that's why we have chosen IPMA; the choice of certification for IPMA for example is based on that

This mode of learning is usually associated with the development of global project management capabilities, the capability to balance control and governance with flexibility and the capabilities to penetrate and develop new markets. The data obtained from the interviews provide evidence that organisations which place an emphasis on contextual learning are more mature in these types of capabilities. In this regard, understanding context or contextual learning can be considered as being a dynamic capability in its own right, which can generate new globally-oriented capabilities.

\section{Research}

This mode is usually associated with the capability to collect required information such as contract laws and legislation in different countries. Examples of organisational capabilities associated with this mode of learning include the capability to enter new markets or engage in international contract management. For example, one of the companies studied while entering a new market realised risks that can arise from the different contract laws they will have to use. Because of that, the company decided to establish a legal department, which contributed to improving its capabilities in global project management and international contract management. Another company studied in the research conducted a big data search and developed fifty new documents related to standards and procurement conditions. From the data, one of the important capabilities of a high maturity company, which is a global development and manufacturing company, is to understand the contexts of different markets in relation to customer requirements and tastes. The company gathers extensive information from different countries, including the different standards and regulations. This capability is also linked with another capability, which is the capability to develop products for different markets with more common elements and less customisation. A manger says: 
You have to make a decision in your design where to differentiate and where to keep the same ... very few products in the world appeal to everyone in the world, take for example the iPhone, I don't know how they do that!

From the above, there is evidence that the company is aware of the need to further develop the capability of developing products that appeal to everyone in the world. Several capabilities are necessary to do that. The capability to do market research and research about standards and regulations is also vital.

The third set of modes are related to organisational wide practices beyond the context or project management, including:

\section{Learning from external sources}

Interviews show that less mature organisations learn more from external sources than internal learning compared to mature ones. External sources of learning in less mature organisations are mostly through learning accomplished though external consultants, whereas mature companies adopt a more systematic benchmarking approach.

\section{Employee feedback}

By this, we mean employees offering feedback on practice or routines for the purpose of improvement; this can manifest in the form of employee suggestions. Employee feedback can be a source of learning and reconfiguration of practice, leading to new capabilities.

\section{Quality management tools}

There is some evidence from the data that the use of quality tools and techniques can offer good avenues for learning. Examples of these include root cause analysis, inspection and value engineering. An example of this mode of learning can be seen in one of the case studies, which is a design and construction company for large infrastructure projects; due to delays in projects, they established a routine for senior managers to regularly visit and inspect their 
construction sites. This new routine opened up many opportunities for learning and troubleshooting and fast problem solving.

\section{Performance management}

Similar to the use of quality tools, there is some evidence from the interviews that performance management frameworks, such as the 'Balanced Scorecard’ (see Kaplan and Norton 1992), can also help in knowledge sharing through learning about the organisational successes and issues. Organisations need to develop a performance management system that helps achieve their strategic objectives and use the right measures and indicators that can lead to better learning. This is in line with the findings of Fouché and Rolstada (2010) where they describe performance measurement in projectised organisations 'as a core process and holistic basis for the control of single project life cycles as well as experience transfer and organisational learning in a longer term multi-project perspective’

\section{Training and education}

This mode is simply the traditional class learning in education or training courses.

The fourth set is related to the prevailing culture of the organisation, including:

\section{Innovative thinking}

Fostering and encouraging innovative thinking can be a rich mode for generating new ideas, especially ideas that do not follow traditional ways of thinking and learning. An example can be seen in Case $\mathrm{H}$, a telecommunications operator, at the time of the financial crash, when businesses were losing and shrinking, this company spotted an opportunity for growth and decided to expand their network to improve service quality and increase market share. One of the interviewees described this situation by saying: 
During the financial crash we decided to invest as we saw an opportunity - everybody else was pulling back... We invested in the infrastructure, you know, networks for better quality and that gave us a competitive advantage, and enabled us to capture the market share.

\section{Leadership led}

In this mode, top management who give direct instructions on the improvements to be made, which are then followed by employees to improve practices, drives learning. This mode is predominantly observed in moderately or less mature organisations where top management are the catalysts and drivers of learning as a means to achieve improvements.

\section{Face-to-face}

Linked to the previous discussion of 'practice to codification' and 'codification to practice', a more commonly used learning mode in practice is face-to-face; this is widely used by mature and moderately mature organisations. Mature organisations seek to find venues for more informal ways for face-to-face interactions to occur between employees, even globally within global teams. This mode of learning was illustrated within the research by practices such as encouraging discussion during review meetings and in-company training. This mode intersects with many other modes and practices of learning, for instance all sorts of meetings can facilitate face-to-face discussions and learning. Among the many benefits of teamwork is that it is also a way of sharing face-to-face knowledge. An interviewee explains:

I think the more powerful one is informal; you need to have people, so we learn from people to people

whilst another manager states:

The project delivery profession was set up as a means of creating informal knowledge exchange opportunities.

The above quotes emanate from participants situated in high maturity organisations and appear to indicate a greater recognition amongst such organisations, than seen in less mature ones, of the need for informal and face-to-face learning modes. 
What is noticeable is that mature and moderately mature organisations show evidence of more modes of learning compared with less mature ones. It is noticeable that less mature organisations have a greater tendency to learn from top management - leadership led learning - and from individuals. Analysis of the interviews shows that maximum learning happens in the more practice-based modes of learning. It also shows that for an organisation to be a mature leaning organisation, they need to invest into finding venues for learning in practice to happen with emphasis on the modes of 'practice to codification' and 'codification to practice'. The challenge here is to maximize the throughput of putting learning into practice. This is of high importance in the management of projects, where the organisations and individuals involved often work under pressure of multiple constraints. Therefore, project management methods and processes need to find new ways to facilitate the maximisation of learning in practice. This may require new practice learning oriented processes or possibly a new knowledge area, yet undefined, in the major project management bodies of knowledge. Such practice oriented learning processes, once recognised and accepted, will lead to new roles for new members of the project team or additional roles for existing team members.

\section{A Learning Capability Model}

The results of this study, which are based on a micro-level perspective, have produced empirical evidence of the preference of a practice-based approach to the understanding and enactment of learning mechanisms in organisations. The main premise is that learning takes place within the sphere of people that are interacting - individuals or groups - with the social structure of the organisation, including the existing knowledge, resulting in a new knowledge base and improved capabilities and business performance. This perspective resonates with the notion of duality of structures proposed by Giddens (1984), a construct he called structuration, which describes how knowledgeable agents draw from social structures in producing and reproducing social systems. Here we refer to the 'knowledgeable agents' in the 
general sense of structuration theory, not to people with greater knowledge in a particular field. In this section, we present a general model for learning capability in organisations.

Figure 3 illustrates the learning capability model. This model does not represent purposeful events of learning in isolation — such as lessons learned discussions — rather it represents a continuous 'learning-in-practice’ phenomenon that occurs before, during and after project delivery. It describes a meta-capability (and not simply a process), in that it encompasses resources, behaviours and processes. We argue here that the actions of the leaders of organisations can foster or hinder this learning capability. Hence, for learning capability to be developed and to function effectively, leaders need to develop and maintain conditions which are cognisant of the internal and external context including stakeholders (Harrington et al 2016).

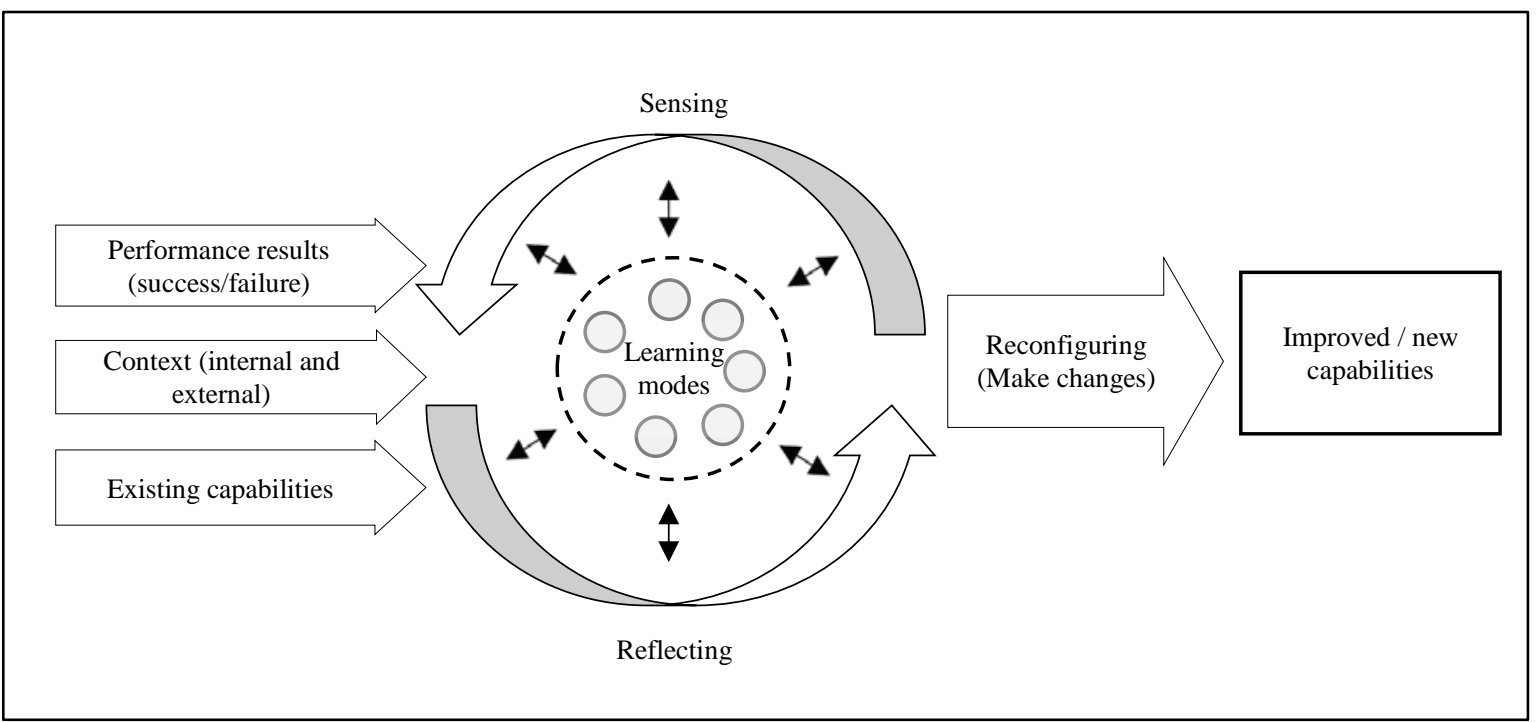

Figure 3- Learning Capability

On the left-hand side of the model are the inputs: performance results, context, and the existing capabilities of the organisation. Results from this research show that organisations mostly learn from failure and usually the highly visible failure events, which attract top management attention. Failures with big impact engage top management in the analysis and 
investigation of reasons and remedies. By contrast, organisations can easily miss the opportunities of learning from successes and good practices in other parts of the organisation. If these successes are not captured and institutionalised across the organisation, this represents a failure to learn. Hughes et al (2016) refer to this as 'Post-mortem process' in their study to investigate factors for project failure. Chipulu et al. (2019) gives a dimensional analysis recognising the non-symmetrical relationship between assessments of success and failure in projects. Here we argue for both success and failure, big or small, as inputs to the learning process. In this way, learning is a continuous process for individuals and organisations.

There is strong evidence from this research that successful and mature organisations put huge emphasis on capturing and understanding the internal and external contexts of learning situations. For example, understanding the market they operate in, including, for instance, its legal, cultural and commercial aspects. In the internal context, organisations need to understand the culture and norms of their organisation. Data shows that mature organisations invest in capturing these elements of context, including the personality traits of the people involved in learning events. A third input to the learning mechanism is the existing capabilities as the aim of learning is always to improve current practices and capabilities.

The central part of the model (learning modes) represents the device of learning including the thirteen learning modes presented in Figure 2, where we argue that learning happens in a reflexive fashion between sensing and reflecting and by interacting with the modes of learning shown in the central part of the figure. The construct of sensing and reflecting is at the heart of Schon's reflective practitioner construct (Schön, 1983), which has been studied extensively in healthcare contexts (see Makridakis et. al. 2019a, b). Later, we argue that being a reflective practitioner is a critical element of an individual's learning skills repertoire that facilitates effective learning in a practice-based learning environment. With regard to the 'sensing' element, we are not only referring to a macro representation of 'sensing market and 
technological opportunities’ as argued by Teece (2007 and 2009), but we extend that to the micro-level representation of an organisation or individual sensing for a need for improvement or change in process or in practice.

Sensing here can come from top management or an individual on the shop floor and needs to be followed by a reflection on activities and experience (Giddens, 1984). Only then can structural changes take place and new or improved capabilities develop. Employees at different levels can formulate ideas and opinions about how the organisation does its business, but only when these ideas are considered and acted upon can they contribute to performance and bottom-line improvements.

This cycle of sensing and reflecting requires mediating policies and cultures that enable it to operate and create value. This can be difficult to establish and typically requires the intervention of senior leadership in creating the essential conditions. In the centre of the learning cycle are the learning modes described in the previous section. We do not argue here that this is an exhaustive or inclusive list of modes, rather these are modes observed in the course of this research and that further studies are desirable in identifying supplementary modes of learning.

The modes of learning are the seeds for learning; they operate in both directions in the cycle of 'sensing' and 'reflecting'. For instance, sensing a need for improvement in a technical aspect can trigger a ‘training and education' mode. In addition, as a result of ‘training and education' employees develop new competencies and can identify or sense a need for change or improvement. 'Reflecting' is the element of acting upon 'sensing' where an individual or a group within the organisation takes steps towards finding solutions or alternatives for possible changes. This leads to the 'Reconfiguring (make changes)' step, where actual changes take 
place in the form of codified improvements or change in the norms and culture, paving the way for improved or new capabilities.

For the above to take place, we identify the following conditions that are required to be in place for an effective learning capability:

1. Leadership commitment

2. Developing and facilitating diverse modes of learning

3. Create opportunities for employees from different parts of the organisation to meet and share knowledge, especially from different parts of the world in the case of multinational companies

4. Balance between rigor and freedom

5. Embedding learning in project management methodologies and processes

As mentioned above, there is a major role for leadership in the development of learning capability in an organisation; this leads all the other four conditions. There is evidence that mature organisations follow diverse learning modes to facilitate maximum learning opportunities. They also seek to establish informal conversations as a powerful learning opportunity for employees to learn from each other. An outcome from this research is the need for balance between rigor in following processes and policies versus freedom of decision making and adaptation. This is of high importance for the proposed model to operate as illustrated in the central part of the diagram. For people to sense, reflect and reconfigure their actions; the right balance needs to be found. An employee who is forced to rigorously follow processes will not sense and reflect effectively. Similarly, too much freedom from very loose processes or a lack of processes, especially as an organisation grows, can lead to chaos. The last condition identified above is to embed processes and roles in project management methodologies to encourage and facilitate the learning process. We argue that this creates a 
need for new learning-based processes and roles that are not yet included within the published project management bodies of knowledge, but which should be considered and certainly be the subject of further research and experimentation.

\section{Conclusions}

This paper argues for the intensification of practice-based learning mechanisms in project delivery. The research identifies thirteen learning modes that are situated at the nucleus of a novel learning capability model. The results suggest that project management governance, methods and processes should seek to embrace new methods and methodologies to enable the maximisation of learning in practice. This may require new, practiced based and more socially oriented processes for learning or possibly a new knowledge area, as yet undefined, in the major project management bodies of knowledge. Such new learning orientated processes, once recognised and accepted, may generate new roles, requiring novel capabilities, for members of the project team and its wider network of stakeholders.

\section{Acknowledgements}

The authors grateful acknowledge the financial support of the Project Management Institute (PMI), this project was undertaken as part of a PMI research award examining the organisational learning in 'strategy-project systems'

\section{References}

Ahern, T., Leavy, B., Byrne, P.J., 2014(a). Complex project management as complex problem solving: a distributed knowledge management perspective. Int. J. Proj. Manag. 32 (8), 1371-1381.

Ahern, T., Leavy, B., Byrne, P.J., 2014 (b). Knowledge formation and learning in the management of projects: A problem solving perspective. Int. J. Proj. Manag. 32 (8), $1423-1431$.

Al-Busaidi, K., \& Olfman, L. (2017). Knowledge sharing through inter-organizational knowledge sharing systems. VINE Journal of Information and Knowledge Management Systems, 47(1), 110-136 
Bosch, O.J.H., Nguyen, N.C., Maeno, T. and Yasui, T. (2013). 'Managing complex issues through evolutionary learning laboratories’, Systems Research, 30, 116-135.

Bourdieu, P. 1977. Outline of a Theory of Practice, Cambridge university press.

Chipulu, M., Ojiako, U., Marshall, A., Williams, T., Bititci, U., Mota, C., Shou, Y., Thomas, A., El Dirani, A., Maguire, S., and Stamati, T. (2019), A dimensional analysis of stakeholder assessment of project outcomes, Production Planning \& Control, In Press

Creswell, J. W., \& Poth, C. N. (2017). Qualitative inquiry and research design: Choosing among five approaches. Sage publications

Davies, A. \& Brady, T. (2016). Explicating the dynamics of project capabilities. International Journal of Project Management, 34(2), 314-327.

Davies, A., Dodgson, M., \& Gann, D. (2016). Dynamic capabilities for complex projects: The case of London Heathrow Terminal 5. Project Management Journal, 47(2), 26-46.

Duffield, S., \& Whitty, S. J. (2015). Developing a systemic lessons learned knowledge model for organisational learning through projects. International Journal of Project Management, 33(2), 311-324.

Easterby-Smith, M. \& Prieto, I. M. 2008. Dynamic Capabilities and Knowledge Management: an Integrative Role for Learning? British Journal of Management, 19

Fouché, D. P., \& Rolstadas, A. (2010). The use of performance measurement as a basis for project control of offshore modification oil and gas projects. Production Planning \& Control, 21(8), 760.

Giddens, A. (1984). The Constitution of Society, Outline of the Theory of Structuration, Cambridge, Polity.

Goffin, K., Koners, U., Baxter, D., \& van, d. H. (2010). Managing Lessons Learned and Tacit Knowledge in New Product Development. Research Technology Management, 53(4), 39-51.

Grundy, T. (1998) Strategy implementation and project management, International Journal of Project Management, Volume 16, Issue 1, February 1998, Pages 43-50

Hartmann, A. and Dorée, A., 2015. Learning between projects: More than sending messages in bottles. International journal of project management, 33(2)

Harrington, T., Singh Srai, J., Kumar, M. and Wohlrab, J. 2016. “Identifying design criteria for urban system 'last-mile' solutions - a multi-stakeholder perspective.” Production Planning \& Control 27(6): 456-476.

John Seely, B. \& Duguid, P. 2001. Knowledge and organization: A social-practice perspective. Organization Science, 12, 198-213.

Kaplan, S and Norton, D. P. (1992). The Balanced Scorecard - Measures that drive performance, Harvard Business Review, Jan-Feb 1992

McClory, S., Read, M., \& Labib, A. (2017). Conceptualising the lessons-learned process in project management: Towards a triple-loop learning framework. International Journal of Project Management, 35(7), 1322-1335

Mainga, W. 2017 "Examining project learning, project management competencies, and project efficiency in project-based firms (PBFs)", International Journal of Managing Projects in Business, Vol. 10 Issue: 3, pp.454-504 
Makridakis, S. Kirkham, R., Wakefield, A., Papadaki, M., Kirkham, J. and Long, L. (2019a) Forecasting, uncertainty and risk; perspectives on clinical decision-making in preventive and curative medicine, International Journal of Forecasting, Volume 35, Issue 2, April-June 2019 pp.659-666

Makridakis, S. Wakefield, A. and Kirkham, R. (2019b) Predicting Medical Risks and Appreciating Uncertainty, Foresight: The International Journal of Applied Forecasting, 52, 28-35

Morris, P and Jamieson, A (2004) Translating corporate strategy into project strategy: realizing corporate strategy through project management, PMI, ISBN:1930699379

Newell, S., \& Edelman, L. F. (2008). Developing a dynamic project learning and cross-project learning capability: Synthesizing two perspectives. Information Systems Journal, 18(6), 567-591.

Nielsen, A. P. 2006. Understanding dynamic capabilities through knowledge management. Journal of Knowledge Management, 10, 59-71.

Nonaka, I. \& Toyama, R. 2002. A firm as a dialectical being: towards a dynamic theory of a firm. Industrial and Corporate Change

Nonaka, I. 1991. The knowledge-creating company. arvard Business Review.

Polanyi, M. 1966. The Tacit Dimension, Doubleday and Company, New York, NY.

Ollus, M., Jansson, K., Karvonen, I., Uoti, M. and Riikonen, H. 2011. “Supporting collaborative project management.” Production Planning \& Control 22 (5-6): 538-553

Orlikowski, W. J. 2002. Knowing in practice: Enacting a collective capability in distributed organizing. Organization Science, 13, 249-273.

Prencipe, A. \& Tell, F. 2001., Inter-project learning: processes and outcomes of knowledge codification in project-based firms, Research Policy, Volume 30, Issue 9, Pages 13731394, ISSN 0048-7333

Regnér, P. 2008. Strategy-as-practice and dynamic capabilities: Steps towards a dynamic view of strategy. Human Relations, 61, 565.

Rolstadas, A. 1994. “Editorial Project management.” Production Planning \& Control 5 (1): p.1

Schön, D. (1983), The Reflective Practitioner: How Professionals Think in Action, Maraca Temple Smith, London

Schiefloe, P.M. (2011), Mennesker og samfunn, Fagbokforlaget, Bergen.

Söderlund, J., 2010. Knowledge entrainment and project management: the case of large-scale transformation projects. International Journal of Project Management 28 (2), 130-141.

Teece, D. 2007. Explicating dynamic capabilities: the nature and microfoundations of (sustainable) enterprise performance. Strategic Management Journal, 28, 1319.

Teece, D. J. 2009. Dynamic capabilities and strategic management: organizing for innovation and growth, GB, Oxford University Press.

Thompson, A. (2005). Getting real value out of lessons databases. Knowledge Management Review, 8(5), 20-23. 
Tsoukas, H. 2009. A Dialogical Approach to the Creation of New Knowledge in Organizations. Organization Science, 20, 941-957.

Tsoukas, H. 2003. Do we really understand tacit knowledge? In: Esterby-Smith, M. A. L. (ed.) Handbook of Organizational Learning and Knowledge. Blackwell, Oxford, UK.

Tsoukas, H. 1996. The firm as a distributed knowledge system : A constructionist approach. Strategic Management Journal, 17, 11-11.

Shokri-Ghasabeh, M., \& Chileshe, N. (2014). Knowledge management: Barriers to capturing lessons learned from Australian construction contractors perspective. Construction Innovation, 14(1), 108-134.

Winter, S. G. 2003. Understanding Dynamic Capabilities. Strategic Management Journal, 24, 991-995.

Whittington, R. 2010. Giddens, Structuration theory and Strategy as Practice. In: Golsorkhi, D., Rouleau, L., Seidl, D. \& vaara, E. (eds.) Cambridge Handbook of Strategy as Practice. Cambridge: Cambridge University Press.

Yin, R. K. 2009. Case Study Research: Design and Methods, Thousand Oaks, CA, Sage Publications.

Zhu, Z. 2006. Nonaka meets Giddens: A critique. Knowledge Management Research \& Practice, 4.

Zollo, M. \& Winter, S. G. 2002. Deliberate learning and the evolution of dynamic capabilities. Organization Science 